TRANSACTIONS OF THE

AMERICAN MATHEMATICAL SOCIETY

Volume 363, Number 1, January 2011, Pages 63-73

S 0002-9947(2010)05095-0

Article electronically published on August 16, 2010

\title{
RELATIVE ISOMETRIC EMBEDDINGS OF RIEMANNIAN MANIFOLDS
}

\author{
MOHAMMAD GHOMI AND ROBERT E. GREENE
}

\begin{abstract}
We prove the existence of $C^{1}$ isometric embeddings, and $C^{\infty}$ approximate isometric embeddings, of Riemannian manifolds into Euclidean space with prescribed values in a neighborhood of a point.
\end{abstract}

\section{INTRODUCTION}

Nash's celebrated theorems in differential geometry [14, 15] and their refinements [6, 2] show that any Riemannian manifold may be isometrically embedded in a Euclidean space. In this paper we obtain some relative versions of these results; i.e., we study the existence and regularity of isometric embeddings whose values have been prescribed in a neighborhood of a point. To state our main results precisely, let $N=N(n, k)$ be the smallest integer such that every $C^{k}$ Riemannian $n$-manifold admits a $C^{k}$ isometric embedding into the Euclidean space $\mathbf{R}^{N}$.

Theorem 1.1. Let $(M, g)$ be a $C^{1 \leq k \leq \infty}$ Riemannian n-manifold, $p \in M$, and $U$ be a neighborhood of $p$. Suppose there exists a $C^{k+1}$ isometric embedding $f: U \rightarrow \mathbf{R}^{m}$, $m>n$. Then there exists a $C^{1}$ isometric embedding $\bar{f}: M \rightarrow \mathbf{R}^{N+m}$, and $a$ neighborhood $V \subset U$ of $p$, with closure $\bar{V}$ diffeomorphic to a ball, such that

$$
\left.\bar{f}\right|_{\bar{V}}=f,
$$

and $\bar{f}$ is $C^{k}$ on $M-\bar{V}$.

Although it can be shown that $\bar{f}$ in the above theorem is somewhat more regular on $\partial V$ than just $C^{1}$, see Proposition 4.1 below, we do not know if in general $\bar{f}$ can be $C^{k}$ on $\partial V$ and therefore $C^{k}$ on all of $M$. Still, we can always achieve this degree of regularity after an arbitrarily small $C^{k}$ perturbation of $g$ near $\partial V$ :

Theorem 1.2. Let $(M, g), p, U$, and $f: U \rightarrow \mathbf{R}^{m}$, be as in Theorem 1.1. Then there exists a neighborhood $V \subset U$ of $p$ and $a C^{k}$ embedding $\bar{f}: M \rightarrow \mathbf{R}^{N+m}$, such that

$$
\left.\bar{f}\right|_{\overline{V-A}}=f,
$$

for any given open neighborhood $A$ of $\partial V$; the pull-back metric $\bar{f}^{*}\langle$,$\rangle is as C^{k}$ close to $g$ as desired; and, $\bar{f}^{*}\langle\rangle=$,$g on M-A$.

Received by the editors April 28, 2008.

2010 Mathematics Subject Classification. Primary 53C42, 53A07.

Key words and phrases. Relative isometric embedding, Riemannian manifold, short map.

The first-named author was supported by NSF Grant DMS-0336455 and CAREER award DMS-0332333.

(C)2010 American Mathematical Society Reverts to public domain 28 years from publication 
In other words, we can construct an arbitrarily fine $C^{k}$ approximate isometric embedding $\bar{f}: M \rightarrow \mathbf{R}^{N}$ which is an (exact) isometric embedding outside of any neighborhood of $\partial V$. Estimates for $N$ in the above results may be obtained from Nash's theorems and their refinements:

Note 1.3. If $k=1$, or $k \geq 3,5, \infty$, in Theorem 1.1, we may set $N=2 n+1, n^{2}+$ $10 n+3,(n+2)(n+3) / 2$, and $\max \{n(n+5) / 2, n(n+3) / 2+5\}$, respectively. The case $k=1$ is given by Nash's theorem [14, Thm. 2]; see also [13, 3. For the cases $k \geq 3$ and $k \geq 5$, see Gromov's book [6, p. 223]. The case $k=\infty$ is due to Günther 7. 8. For low dimensional estimates and other references, see [9]. A nice survey of Nash's isometric theorems and related results is given in [2].

We should point out that the above theorems are optimal in the following sense:

Note 1.4. It is not possible in general to require that the extension $\bar{f}$ in Theorem 1.1 coincide with the prescribed embedding $f$ over the entire domain $U$, or all compact subsets of it. The simplest counterexample is given by the circle $\mathbf{S}^{1}$ : let $U$ be a connected open subset of $\mathbf{S}^{1}$ of length greater than $\pi$ and $f: U \rightarrow \mathbf{R}$ be an isometric mapping. Then $f$ cannot be extended isometrically to all of $\mathbf{S}^{1}$, because any such extension would have total length greater than $2 \pi$. Similarly, one can construct counterexamples of every dimension by using the flat tori $T^{n}=\mathbf{S}^{1} \times \cdots \times \mathbf{S}^{1}$. Perturbing the metric of these tori will yield nonflat counterexamples as well.

Furthermore, the dimension $N+m$ in our main results is not extravagant:

Note 1.5. If a Riemannian manifold $M$ has a global isometric embedding in $\mathbf{R}^{m}$, there may still exist a local isometric embedding $f$ of a neighborhood $U$ of $M$ such that $f$ restricted to no open subset $V$ of $U$ may be extended to a global isometric embedding of $M$ in $\mathbf{R}^{m}$. In particular, the occurrence in Theorem 1.1 of the higher dimensions $m+N$ for the ambient space is not in general superfluous. Such examples arise from situations wherein the embedding of $M$ is globally but not locally rigid. Consider for instance the well-known surfaces of revolution in $\mathbf{R}^{3}$ which are of constant Gauss curvature 1 but which have mean curvature which is not constant on any open subset; see for instance [12] or [16] (these surfaces are of course not complete). The Gauss curvature being 1 implies that every point has a neighborhood isometric to an open subset of the standard unit sphere $\mathbf{S}^{2}$ in $\mathbf{R}^{3}$, but the mean curvature property indicated means that no open subset of these surfaces can be congruent to an open subset of $\mathbf{S}^{2}$; these surfaces are in this sense everywhere locally nonspherical. On the other hand, the well-known rigidity theorem of CohnVossen, which applies to surfaces with as little regularity as $C^{2}$ by the proof of Herglotz [10, shows that the only isometric embedding of $\mathbf{S}^{2}$ in $\mathbf{R}^{3}$ is $\mathbf{S}^{2}$ itself up to a rigid motion. Hence no open subset of a nonspherical surface of revolution of constant Gauss curvature 1 can be extended to a $C^{2}$ global isometric embedding of $\mathbf{S}^{2}$ in $\mathbf{R}^{3}$ (this also follows from Hopf's maximum principle for elliptic equations; see [19 and [18, p. 211]). Indeed, the rigidity theorem of Pogorelov [17, p. 167], who generalized Cohn-Vossen's result, shows that such an extension is not possible as a convex embedding even when no additional regularity is required of the extension, i.e., when the surface is only required to be the boundary of a compact convex set. Furthermore, local nonrigidity of convex surfaces shows that these examples are a special case of a much more general process: any open subset of a closed convex surface, whose complement has nonempty interior, admits a nontrivial isometric 
deformation [17, p. 172]. This will yield a large class of examples of the type indicated above via Pogorelov's global rigidity theorem.

A $C^{2}$ immersion $f: M \rightarrow \mathbf{R}^{N}$ is said to be free if the set of all its first and second derivatives is linearly independent.

Note 1.6. When $f$ in Theorem 1.1 is free, one might be able to show that $\bar{f}$ is $C^{k}$ everywhere, since then Nash's implicit function theorem applies. In this case, it might also be possible to replace $N+m$ by $\max \{N, m\}$, and this may already be implicit in works of Nash [14] or Gromov [6]. But for $f$ to be free we must have $m \geq n+n(n+1) / 2$ (which may constitute a heavy price, or be unwarranted).

For an application of Theorem 1.2 we refer the reader to [1] where it is proved that the standard sphere $\mathbf{S}^{2} \subset \mathbf{R}^{3} \subset \mathbf{R}^{18}$ bounds infinitely many distinct toplogical types of $C^{\infty}$ positively curved compact submanifolds.

While we are not aware of any previous work on the relative isometric problem considered here, it should be mentioned that there is a paper of Jacobowitz [11] where he studies the problem of locally extending the isometric embedding of a codimension-one submanifold to its ambient space. Gromov's book [6, §3.1.6] also contains a number of other local extension results.

Our proofs of Theorems 1.1 and 1.2, which are presented in Section 3, employ a variation of a Cartesian product technique used in Nash's work [15, Part D], see also [5], to piece together the desired isometric embedding from certain other maps. One of these maps, which we construct in Section 2, is a short mapping $f_{1}: M \rightarrow \mathbf{R}^{m}$ which sends most of $M$ to the origin, preserves $f$ on a neighborhood $V$ of $p$, and is strictly short everywhere else. We then let $g_{1}:=f_{1}^{*}\langle$,$\rangle be the induced$ symmetric bilinear form on $M\left(g_{1}\right.$ vanishes on most of $M$, but $g_{1}=g$ on $\left.V\right)$. Next we let $g_{2}:=g-g_{1}$ be the complementary form on $M$, and show that there exists a $C^{1}$ mapping $f_{2}: M \rightarrow \mathbf{R}^{N}$ such that $f_{2}$ maps $V$ to the origin, and $f_{2}^{*}\langle\rangle=,g_{2}$. Then $\bar{f}: M \rightarrow \mathbf{R}^{N+m}$ given by $\bar{f}:=\left(f_{1}, f_{2}\right)$ is the desired embedding, for it induces the metric $g_{1}+g_{2}=g$ on $M$ and coincides with $f_{1}=f$ on $V$. Theorem 1.2 will be proved by a perturbation of $g_{2}$, which will ensure that the corresponding map $f_{2}$ is smooth. The precise details for these arguments are described in the next two sections. The regularity of the map $f_{2}$, which determines the regularity of $\bar{f}$, is further studied in Section 4 , and the remaining questions are summarized in Section 5 .

\section{Relative SHORT MAPS}

A metric in this paper always means a positive definite symmetric bilinear form. We say that $(M, g)$ is a $C^{k}$ Riemannian manifold provided that $M$ is a $C^{\infty}$ manifold with a $C^{k}$ metric $g$. Recall that any $C^{1}$ map $f: M \rightarrow \mathbf{R}^{n}$ induces a (possibly degenerate) symmetric bilinear form $f^{*}\langle$,$\rangle on M$ given by

$$
\left(f^{*}\langle,\rangle\right)_{p}(X, Y):=\left\langle d f_{p}(X), d f_{p}(Y)\right\rangle
$$

for all $p \in M$ and $X, Y \in T_{p} M$, where $d f$ denotes the differential map of $f$, and $\langle$,$\rangle is the standard metric on \mathbf{R}^{n}$. We say that $f$ is short provided that

$$
f^{*}\langle,\rangle \leq g
$$

i.e., $\left(f^{*}\langle,\rangle\right)_{p}(X, Y) \leq g_{p}(X, Y)$ for all $p \in M$ and $X, Y \in T_{p} M$. If the inequality is strict at some point $p$, we say that $f$ is strictly short at $p$, and if equality holds everywhere, then $f$ is an isometric map. 
As we mentioned earlier, the first step towards proving Theorems 1.1 and 1.2 is to show that there exists a (relative) short mapping of $M$ into $\mathbf{R}^{n}$ which coincides with the prescribed values given by $f$ near the point $p$. Further, we require that this mapping be strictly short everywhere else. This is the content of Proposition 2.3 below, which is the main result of this section. First we need the following elementary fact:

Lemma 2.1. For any $\epsilon>0$ and $0<c<1$, there exists an $0<\epsilon^{\prime}<\epsilon$ and a $C^{\infty}$ concave function $\theta:[0, \infty) \rightarrow[0, \infty)$ such that

(1) $\theta(t)=t$ on $\left[0, \epsilon^{\prime}\right]$,

(2) $\theta(t)<t$ on $\left(\epsilon^{\prime}, \infty\right)$,

(3) $\theta(t)<$ ct on $(\epsilon, \infty)$

(4) $\theta^{\prime}(t)>0$ on $[0, \infty)$

Proof. We construct $\theta$ by smoothing the piecewise linear concave function $\bar{\theta}$ determined by the lines $y=t$ and $y=c(t+\epsilon) / 2$, i.e., set $\bar{\theta}(t):=t$ on $(-\infty, a]$, and $\bar{\theta}(t):=c(t+\epsilon) / 2$ on $[a, \infty)$, where $a=\epsilon /(2-c)$; see Figure 1.

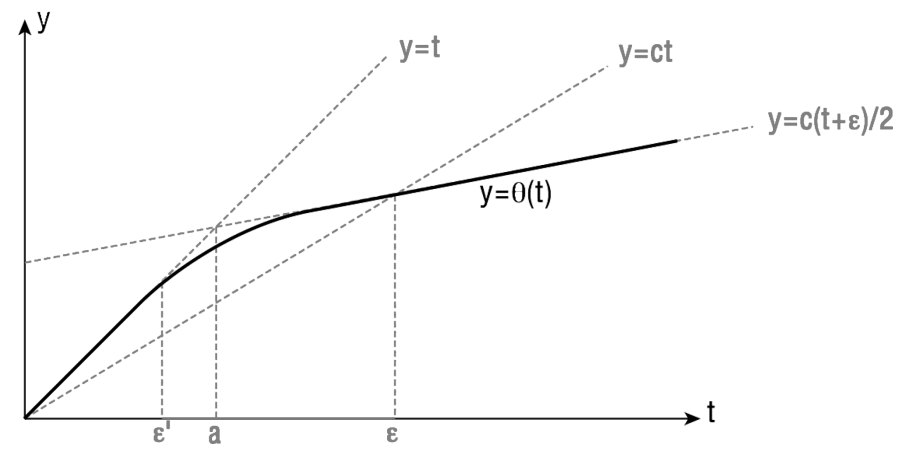

Figure 1

Then we define $\theta$ as the moving average, or convolution $\bar{\theta} * \eta$, where $\eta: \mathbf{R} \rightarrow[0, \infty)$ is a $C^{\infty}$ function with $\int_{\mathbf{R}} \eta(t) d t=1, \eta(t)=\eta(-t)$, and $\operatorname{supp} \eta \subset[-\delta, \delta]$, where $0<\delta<\min \{a, \epsilon-a\}$. Since $\bar{\theta}$ is concave, it follows that $\theta$ is concave as well. It is also easy to check that $\theta(t)=t$ at all points $t$ such that $\bar{\theta}$ is linear on $[t-\delta, t+\delta]$; see [4]. In particular, $\theta(t)=t$ on $\left[0, \epsilon^{\prime}\right]$ where $\epsilon^{\prime}=a-\delta$. Further, $\theta(t)<t$ on $\left(\epsilon^{\prime}, \infty\right)$, since $\bar{\theta}(t) \leq t$, and for any $t \in\left(\epsilon^{\prime}, \infty\right), \bar{\theta}(s)<s$ for some $s \in(t-\delta, t+\delta)$. Furthermore, $\theta(t)=\bar{\theta}(t)=c / 2<c$ on $(\epsilon, \infty)$, since $\bar{\theta}$ is linear on $(\epsilon-\delta, \infty)$. Finally, since $\theta$ is concave, $\theta^{\prime \prime} \leq 0$, so $\theta^{\prime}$ is nonincreasing, but $\theta^{\prime}=c / 2$ on $(\epsilon, \infty)$; therefore, $\theta^{\prime} \geq c / 2>0$ everywhere.

For any set $X, \bar{X}$ denotes the closure of $X$, and $B_{r}(p)$ stands for an open ball of radius $r>0$. The last lemma yields:

Lemma 2.2. For any $p \in \mathbf{R}^{n}, \epsilon>0$, and $0<c<1$, there exist an $0<\epsilon^{\prime}<\epsilon$ and a $C^{\infty}$ short embedding $h: \mathbf{R}^{n} \rightarrow \mathbf{R}^{n}$ such that $h$ is the identity on $\bar{B}_{\epsilon^{\prime}}(p) \subset \mathbf{R}^{n}, h$ is strictly short on $\mathbf{R}^{n}-\bar{B}_{\epsilon^{\prime}}(p)$, and $h^{*}\langle\rangle,\left\langle c\langle\right.$,$\rangle on \mathbf{R}^{n}-\bar{B}_{\epsilon}(p)$. 
Proof. After a rigid motion we may assume that $p=0$. Let $\theta$ be given by Lemma 2.1. and set

$$
h(x):=\frac{\theta(\|x\|)}{\|x\|} x .
$$

We claim that this is the desired map. First note that, by part (1) of Lemma 2.2. $h$ is the identity on $\bar{B}_{\epsilon^{\prime}}(0)$. In particular $h$ is $C^{\infty}$ near the origin 0 of $\mathbf{R}^{n}$. This together with the smoothness of $\theta$ and the norm \|\| on $\mathbf{R}^{n}-\{0\}$ shows that $h$ is $C^{\infty}$ everywhere.

Next we show that $h$ is a $C^{\infty}$ embedding. First note that $\theta$ is one-to-one by item (4) of Lemma 2.2 above. This easily yields that $h$ is one-to-one. It remains then to check that $d h$ is nondegenerate: a routine computation shows that, for any $q \in \mathbf{R}^{n}-\{0\}$ and $X \in T_{q} \mathbf{R}^{n}$,

$$
d h_{q}(X)=\frac{\theta(\|q\|)}{\|q\|} X+\left(\theta^{\prime}(\|q\|)-\frac{\theta(\|q\|)}{\|q\|}\right) \frac{\langle X, q\rangle}{\|q\|^{2}} q .
$$

So $d h_{q}(X)=0$ only when $X=\lambda q$ or $d h_{q}(X)=\lambda d h_{q}(q)$. But $d h_{q}(q)=\theta^{\prime}(\|q\|) q \neq$ 0 , since $\theta^{\prime}>0$, by part (4) of Lemma 2.2. Thus $d h_{q}$ is nondegenerate for all $q \in \mathbf{R}^{n}-\{0\}$. Hence, since $d h_{0}$ is the identity, $d h$ is nondegenerate everywhere.

Now we verify that $h$ is strictly short outside $\bar{B}_{\epsilon^{\prime}}(0)$. For any $q \in \mathbf{R}^{n}-\{0\}$, let $E_{i}, i=1, \ldots, n$ be an orthonormal basis for $\mathbf{R}^{n}$ with $X_{n}:=q /\|q\|$. Then (2.1) yields that

$$
d h_{q}\left(E_{i}\right)= \begin{cases}\frac{\theta(\|q\|)}{\|q\|} E_{i}, & \text { if } i<n \\ \theta^{\prime}(\|q\|) E_{i}, & \text { if } i=n\end{cases}
$$

So for any pair of vectors $X=\sum_{i=1}^{n} X^{i} E_{i}, Y=\sum_{i=1}^{n} Y^{i} E_{i}$ in $\mathbf{R}^{n}$,

$$
\left\langle d h_{q}(X), d h_{q}(Y)\right\rangle=\left(\frac{\theta(\|q\|)}{\|q\|}\right)^{2} \sum_{i=1}^{n-1} X^{i} Y^{i}+\left(\theta^{\prime}(\|q\|)\right)^{2} X^{n} Y^{n} .
$$

Further recall that since $\theta$ is concave (i.e., $\left.\theta^{\prime \prime} \leq 0\right), \theta^{\prime}$ is nonincreasing; therefore,

$$
\theta^{\prime}(t)=\inf _{[0, t]} \theta^{\prime} \leq \frac{1}{t} \int_{0}^{t} \theta^{\prime}(s) d s=\frac{\theta(t)}{t} .
$$

So (2.2) yields that

$$
\left(h^{*}\langle,\rangle\right)_{q}(X, Y) \leq\left(\frac{\theta(\|q\|)}{\|q\|}\right)^{2}\langle X, Y\rangle .
$$

This shows, via part (2) of Lemma 2.2, that $h$ is strictly short on $\mathbf{R}^{n}-B_{\epsilon^{\prime}}(0)$. Finally, (2.3), together with part (3) of Lemma2.2 also shows that $h^{*}\langle\rangle,\left\langle c^{2}\langle\rangle<\right.$, $c\langle$,$\rangle on \mathbf{R}^{n}-\bar{B}_{\epsilon}(0)$, which completes the proof.

Now we are ready to prove the main result of this section:

Proposition 2.3. Let $M, p, U$, and $f$ be as in Theorem 1.1. Then there exist open neighborhoods $V, W$ of $p$ with closures diffeomorphic to balls, $\bar{V} \subset \bar{W} \subset U$, and a $C^{k+1}$ short map $\sigma: M \rightarrow \mathbf{R}^{m}$ such that $\sigma=f$ on $\bar{V}, \sigma$ is strictly short on $M-\bar{V}$, is an embedding on $W$, and maps $M-W$ to a point not on $f(\bar{V})$.

Proof. Since $m>n$, we may assume, after a rigid motion, that there exists an open neighborhood $W$ of $p$, with closure $\bar{W} \subset U$ diffeomorphic to a ball, such that no line emanating from the origin intersects $f(W)$ more than once (i.e., $f(W)$ is a 
radial graph). Let $\lambda: U \rightarrow \mathbf{R}$ be a $C^{k+1}$ function such that $0 \leq \lambda \leq 1, \lambda>0$ on $W, \lambda \equiv 0$ on $U-W$, and $\lambda \equiv 1$ on an open neighborhood of $p$. Choose $\epsilon>0$ small enough so that $A:=f^{-1}\left(B_{\epsilon}(f(p))\right) \subset \lambda^{-1}(1)$; for any $0<\epsilon^{\prime} \leq \epsilon, f^{-1}\left(\overline{B_{\epsilon^{\prime}}(f(p))}\right)$ is diffeomorphic to a ball, and for any point $q \in W-\lambda^{-1}(1)$, the line segment connecting $f(q)$ to the origin is disjoint from $B_{\epsilon}(f(p))$; see Figure 2 .

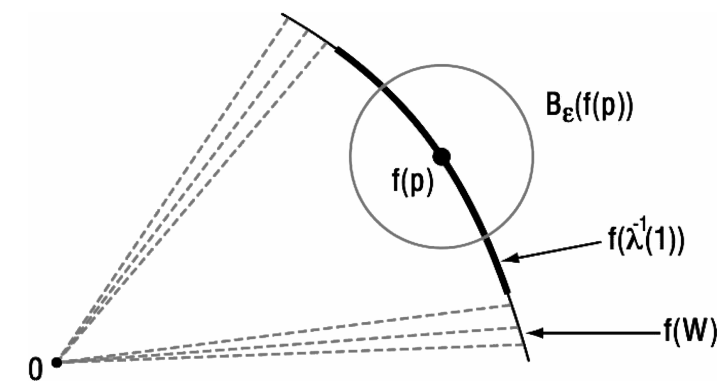

FIGURE 2

Define $\tilde{f}: M \rightarrow \mathbf{R}^{m}$ by $\widetilde{f}(q):=\lambda(q) f(q)$ on $U$, and $\widetilde{f}(q):=0$ on $M-U$. Note that $\tilde{f}$ is $C^{k+1}$ since $\lambda \equiv 0$ on $U-W$. Apply Lemma 2.2 with $p:=f(p)$ (and $\epsilon=\epsilon)$ to obtain the short mapping $h: \mathbf{R}^{n} \rightarrow \mathbf{R}^{n}$. We claim that we may choose $c$ in Lemma 2.2 small enough so that

$$
\sigma:=h \circ \tilde{f}
$$

is the desired map. First note that, since $f(W)$ is a radial graph, and $\lambda>0$ on $W, \sigma$ is one-to-one on $W$; further, since $h$ is the identity precisely on some small ball $\bar{B}^{\prime}:=\overline{B_{\epsilon^{\prime}}(f(p))}$, and $\tilde{f}=f$ on $A \supset \bar{V}:=f^{-1}\left(\overline{B^{\prime}}\right)$, we have $\sigma=f$ on $\bar{V}$, for any choice of $c$. It remains then to show that $\sigma$ is strictly short on $M-\bar{V}$, for sufficiently small $c$.

To find this $c$ note that, for any choice of $c, \sigma$ is strictly short on $A-\bar{V}$, since $\tilde{f}$ is short on $A$, and $h$ is strictly short on $\mathbf{R}^{n}-\bar{B}^{\prime} \supset \widetilde{f}(A-\bar{V})$. The fact that $\widetilde{f}(A-\bar{V}) \subset \mathbf{R}^{n}-\bar{B}^{\prime}$ is due to the assumption at the end of the first paragraph that for any point $q \in W-\lambda^{-1}(1)$, the line segment connecting $f(q)$ to the origin is disjoint from $B_{\epsilon}(f(p))$.

Thus it remains to find $c$ so that $\sigma$ is strictly short on $M-A$. To this end, let $\left|g_{p}\right|$ denote the norm of the metric $g$ at $p \in M$, i.e., $\left|g_{p}\right|:=\sup g_{p}(X, X)$, where $X$ ranges over the unit vectors of the tangent space $T_{p} M$. Note that if $g^{\prime}$ is any other metric on $M$, then $g_{p}<g_{p}^{\prime}$ if and only if $\left|g_{p}\right|<\left|g_{p}^{\prime}\right|$. Next observe that

$$
\sup _{p \in M-A} \frac{\left|\tilde{f}_{p}^{*}\langle,\rangle\right|}{\left|g_{p}\right|}=\sup _{p \in \bar{W}-A} \frac{\left|\tilde{f}_{p}^{*}\langle,\rangle\right|}{\left|g_{p}\right|}=: K<\infty ;
$$

the first equality is due to the fact that $\tilde{f}$ is constant on $M-W$, which implies that $(\widetilde{f})^{*}$ vanishes there, and finiteness of $K$ follows from compactness of $\bar{W}-A$. So we conclude that

$$
\tilde{f}^{*}\langle,\rangle<K g
$$


on $M-A$. Now, if we set $c<1 / K$ in Lemma 2.2, it follows that

$$
\sigma^{*}\langle,\rangle=\widetilde{f}^{*} h^{*}\langle,\rangle<\frac{1}{K} \widetilde{f}^{*}\langle,\rangle<g
$$

on $M-A$. So $\sigma$ is strictly short on $M-A$.

\section{Proof of Theorems 1.1 and 1.2}

Before proving our main results, we need only one more fact:

Lemma 3.1. Let $M$ be a $C^{\infty}$ manifold, and $V \subset M$ be a connected open subset with compact closure $\bar{V}$ and $C^{\infty}$ boundary $\partial V$. Suppose there exists a $C^{k}$, symmetric bilinear form $g$ on $M$ which is positive definite on $M-\bar{V}$ and is identically zero on $\bar{V}$, and let $f: M-\bar{V} \rightarrow \mathbf{R}^{N}$ be a $C^{k}$ isometric embedding. Then there exists a point $p \in \mathbf{R}^{N}$ such that $\bar{f}: M \rightarrow \mathbf{R}^{N}$ defined by $\bar{f}=f$ on $M-\bar{V}$ and $\bar{f}=p$ on $\bar{V}$, is $C^{1}$ on $M$ and $C^{k}$ on $M-\partial V$.

Proof. First we describe how the point $p$ is to be found. Let $h$ be an arbitrary Riemannian metric on $M$, and let $V_{i}$ be a sequence of nested open neighborhoods of $\partial V$, which lie within an $h$-distance $1 / i$ of $\partial V$. Then, for $i$ sufficiently large, $g<h$ on $A_{i}$, since $g$ vanishes on $\partial V$. Indeed, $g<\lambda_{i} h$ on $A_{i}$, where $\lambda_{i}$ is a sequence of positive numbers converging to 0 . So the diameter of $A_{i}$ with respect to $g$ becomes arbitrarily small as $i$ grows larger, since

$$
\operatorname{diam}_{g}\left(A_{i}\right) \leq \lambda_{i} \operatorname{diam}_{h}\left(A_{i}\right) \leq \lambda_{i}\left(\frac{2}{i}+\operatorname{diam}_{h}(\partial V)\right) .
$$

Consequently, the $f\left(A_{i}\right)$ are contained in a sequence of nested closed balls of radii $\operatorname{diam}_{g}\left(A_{i}\right)$ in $\mathbf{R}^{N}$. There is only one point common to all these balls, which we choose as our $p$. This immediately yields that $\bar{f}$ is continuous.

Next we show that $\bar{f}$ is $C^{1}$. To see this, note that, since $\partial V$ is $C^{\infty}$, we may identify, via a diffeomorphism, a neighborhood $W$ of any point of $\partial V$ with $\mathbf{R}^{n}$, where $n=\operatorname{dim}(M)$, so that $W \cap V$ is identified with the lower half-space $x_{n}<0$. Let $g_{i j}$ be the coefficients of $g$ with respect to these coordinates. Then $\left\langle\partial_{i} \bar{f}, \partial_{j} \bar{f}\right\rangle=g_{i j}$ on the complement of the plane $\Pi$ given by $x_{n}=0$. So

$$
\lim _{x \rightarrow \Pi}\left\|\bar{\partial}_{i} f(x)\right\|^{2}=\lim _{x \rightarrow \Pi} g_{i i}(x)=0
$$

since $g$, and consequently $g_{i j}$, vanish on the $\Pi$. So, to show that $\bar{f}$ is $C^{1}$ it suffices to check that $\bar{f}$ is differentiable on $\Pi$ and the $\partial_{i} \bar{f}$ vanish there. To see this, note that for $i=1, \ldots, n-1$ we have $\partial_{i} \bar{f}=0$ on $\Pi$ since $\bar{f}$ is constant there. Further, since $\bar{f}$ vanishes on $\Pi$, l'Hôpital's rule and (3.1) yield that:

$$
\partial_{n} \bar{f}\left(x_{1}, \ldots, x_{n-1}, 0\right)=\lim _{t \rightarrow 0} \frac{\bar{f}\left(x_{1}, \ldots, x_{n-1}, t\right)}{t}=\lim _{t \rightarrow 0} \partial_{n} \bar{f}\left(x_{1}, \ldots, x_{n-1}, t\right)=0 .
$$

Finally, since $\bar{f}$ is $C^{k}$ on $M-\bar{V}$ and is constant on $V, \bar{f}$ is $C^{k}$ on $M-\partial V$.

Now we are ready to prove our main results:

Proof of Theorem 1.1. Let $V, W$, and $\sigma$ be as in Proposition 2.3, and set $f_{1}:=\sigma$. Then $g_{1}:=f_{1}^{*}\langle$,$\rangle is a C^{k}$ metric on $W$, since $f_{1}$ is $C^{k+1}$. Further note that $g_{1}$ vanishes on $M-W$ since $f_{1}$ is constant there. Next set $g_{2}:=g-g_{1}$ (where $g$ is the metric on $M$ ). Then, since $g>g_{1}$ on $M-\bar{V}$, and $g$ and $g_{1}$ are both $C^{k}$, it follows that $g_{2}$ is a $C^{k}$ metric on $M-\bar{V}$. Thus, by Lemma 3.1 there exists a $C^{1}$ mapping 
$f_{2}: M \rightarrow \mathbf{R}^{N}$, such that $f_{2}$ is a $C^{k}$ isometric embedding on $M-\bar{V}$, with respect to $g_{2}$, and maps $\bar{V}$ to a point, say the origin. Now define $\bar{f}: M \rightarrow \mathbf{R}^{N+m}$ by

$$
\bar{f}:=\left(f_{1}, f_{2}\right) .
$$

Then $\bar{f}=f_{1}=f$ on $\bar{V}$. Further, $\bar{f}$ is a $C^{1}$ immersion, and its induced metric on $M$ is $g_{1}+g_{2}=g$. So $\bar{f}$ is an isometric immersion. Furthermore, since $f_{1}$ is $C^{k}$ everywhere, and $f_{2}$ is $C^{k}$ on $M-\partial V$, it follows that $\bar{f}$ is $C^{k}$ on $M-\partial V$. It only remains to check that $\bar{f}$ is one-to-one, which would complete the proof. Note that since $f_{1}$ is one-to-one on $W$ and $f_{2}$ is one-to-one on $M-\bar{V}$, it follows that $\bar{f}$ is one-to-one on $W$ and on $M-\bar{V}$. So we just need to show that $f(M-W)$ is disjoint from $f(\bar{V})$. But $f_{1}(\bar{V})$ is disjoint from the origin, whereas $f_{1}(M-W)$ is the origin by construction of $f_{1}$ (Proposition 2.3). So $\bar{f}$ is the desired mapping.

Proof of Theorem 1.2. Let $V, W, g_{1}, g_{2}$, and $f_{1}$ be as in the proof of Theorem 1.1. Let $V^{\prime}$ be an open neighborhood of $p$ diffeomorphic to a ball with $\overline{V^{\prime}} \subset V$. There exists a $C^{\infty}$ map $\theta: M \rightarrow M$ such that $\theta\left(\overline{V^{\prime}}\right)=p$ and $\theta: M-\overline{V^{\prime}} \rightarrow M-\{p\}$ is a diffeomorphism. Note that $\theta$ induces a symmetric bilinear form $\theta_{*} g_{2}$ on $M$ by defining $\theta_{*} g_{2}$ to be the push-forward of $g_{2}$ via $\theta$ on $M-\{p\}$, i.e.,

$$
\left(\theta_{*} g_{2}\right)_{q}(X, Y):=\left(g_{2}\right)_{\theta^{-1}(q)}\left(d \theta_{q}^{-1}(X), d \theta_{q}^{-1}(Y)\right),
$$

for all $q \in M-\{p\}$, and setting $\left(\theta_{*} g_{2}\right)_{p}:=0$. Further note that $\theta_{*} g_{2}$ is $C^{k}$ since it vanishes on $\theta(V)$, which is an open neighborhood of $p$.

Let $h$ be any $C^{\infty}$ Riemannian metric on $M$, and $\phi: M \rightarrow \mathbf{R}$ be any $C^{\infty}$ nonnegative function with compact support which contains $\theta(V)$. Then $\theta_{*} g_{2}+\epsilon \phi h$ is a $C^{k}$ Riemannian metric on $M$ for any $\epsilon>0$. Thus there exists a $C^{k}$ isometric embedding $f_{2}^{\epsilon}: M \rightarrow \mathbf{R}^{N}$ with respect to this metric. After a translation, we may assume that $f_{2}^{\epsilon}(p)=0$. Next let $g_{2}^{\epsilon}$ be the pull-back of $\theta_{*} g_{2}+\epsilon \phi h$ via $\theta$, i.e., set

$$
g_{2}^{\epsilon}:=\theta^{*}\left(\theta_{*} g_{2}+\epsilon \phi h\right)=g_{2}+\epsilon \theta^{*}(\phi h) .
$$

Note that $g_{2}^{\epsilon}$ is a $C^{k}$ symmetric bilinear form on $M$ which is positive definite on $M-\overline{V^{\prime}}$ and vanishes on $\overline{V^{\prime}}$.

Define $\bar{f}_{2}^{\epsilon}: M \rightarrow \mathbf{R}^{N}$ by

$$
\bar{f}_{2}^{\epsilon}:=f_{2}^{\epsilon} \circ \theta \text {. }
$$

Then $\bar{f}_{2}^{\epsilon}$ is a $C^{k}$ mapping which is an isometric embedding on $M-\overline{V^{\prime}}$ with respect to $g_{2}^{\epsilon}$, and $\bar{f}_{2}^{\epsilon}\left(\overline{V^{\prime}}\right)=0$. Next define $\bar{f}^{\epsilon}: M \rightarrow \mathbf{R}^{N+m}$ by

$$
\bar{f}^{\epsilon}:=\left(f_{1}, \bar{f}_{2}^{\epsilon}\right) .
$$

This is a $C^{k}$ isometric immersion with respect to the metric

$$
g^{\epsilon}:=g_{1}+g_{2}^{\epsilon}=g+\epsilon \theta^{*}(\phi h) .
$$

Further, $\bar{f}^{\epsilon}=f_{1}=f$ on $V^{\prime}$. Choosing $\epsilon$ sufficiently small, we can make sure that $g^{\epsilon}$ is as $C^{k}$ close to $g$ as we may desire. Furthermore, similar to the end of the proof of Theorem 1.1 it can be shown that $\bar{f}^{\epsilon}$ is one-to-one, which yields that it is an embedding.

Finally, note that if $A$ is any open neighborhood of $\partial V$, then we may assume that $\theta$ is the identity and $\phi$ vanishes outside $A \cup V$. This implies that $\theta^{*}(\phi h)$ vanishes outside $A \cup V$, so $g^{\epsilon}=g$ outside $A \cup V$. Furthermore, recall that $g_{2}^{\epsilon} \equiv 0$ on $V^{\prime}$, so $g^{\epsilon}=g_{1}$ on $V^{\prime}$, but $g_{1}=g$ on $V \supset V^{\prime}$ by construction. So $g^{\epsilon}=g$ on $V^{\prime}$. We may choose $V^{\prime}$ so large that $\partial V^{\prime} \subset A$. Then $g=g^{\epsilon}$ outside of $A$. 


\section{More Regularity}

Here we show that $\bar{f}$ in Theorem 1.1 has somewhat more regularity than has been mentioned there. In particular, if $(M, g)$ is $C^{\infty}$ and $\gamma:(-1,1) \rightarrow M$ is any $C^{\infty}$ curve which is a transversal to $\partial V$, then $\bar{f} \circ \gamma$ is $C^{\infty}$. More generally, we have:

Proposition 4.1. Let $\bar{f}$ be as in Theorem 1.1, or Lemma 3.1, and suppose that $k \geq 2 \ell$. Then for any $C^{2 \ell}$ curve $\gamma:(-1,1) \rightarrow M$, which is a transversal to $\partial V$, $\left(\partial_{i} \bar{f}\right) \circ \gamma$ is $C^{\ell}$, with respect to any system of local coordinates. In particular $\bar{f} \circ \gamma$ is $C^{\ell+1}$.

This follows quickly from the following elementary observation:

Lemma 4.2. Let $h:(-1,1) \rightarrow \mathbf{R}^{N}$ be a continuous map. Suppose that $h \equiv 0$ on $(-1,0], h$ is $C^{\ell}$ on $(0,1)$, and $\|h\|^{2}$ is $C^{2 \ell}$ everywhere. Then $h$ is $C^{\ell}$.

Proof. We proceed by induction on $\ell$. First suppose that $\ell=1$. Note that if $h^{\prime}(0)$ exists, then $h^{\prime}(0)=0$, since $h$ is contant on $(-1,0]$. Further, by l'Hôpital's rule,

$$
h^{\prime}(0)=\lim _{t \rightarrow 0} \frac{h(t)-h(0)}{t}=\lim _{t \rightarrow 0} h^{\prime}(t) .
$$

Thus to show that $h$ is $C^{1}$ it suffices to check that $\lim _{t \rightarrow 0} h^{\prime}(t)=0$. To see this, note that all derivatives of $\|h\|^{2}$ have to vanish at 0 . Thus

$$
\left\|\lim _{t \rightarrow 0} h^{\prime}(t)\right\|^{2}=\left\|\lim _{t \rightarrow 0} \frac{h(t)}{t}\right\|^{2}=\lim _{t \rightarrow 0} \frac{\|h(t)\|^{2}}{t^{2}}=\lim _{t \rightarrow 0} \frac{\left(\|h(t)\|^{2}\right)^{\prime \prime}}{2}=0 .
$$

Next suppose that the lemma holds for $\ell$. Then we need to show that $h$ is $C^{\ell+1}$. Note that, similar to the case for $\ell=1$, we have

$$
h^{(\ell+1)}(0)=\lim _{t \rightarrow 0} \frac{h^{(\ell)}(t)-h^{(\ell)}(0)}{t}=\lim _{t \rightarrow 0} h^{(\ell+1)}(t) .
$$

So, again we just need to verify that $\lim _{t \rightarrow 0} h^{(\ell+1)}(t)=0$. The computation is a straightforward generalization of the one given earlier:

$$
\begin{aligned}
\left\|\lim _{t \rightarrow 0} h^{(\ell+1)}(t)\right\|^{2} & =\left\|\lim _{t \rightarrow 0} \frac{h^{(\ell)}(t)}{t}\right\|^{2} \\
& =\left\|\lim _{t \rightarrow 0} \frac{\ell ! h(t)}{t^{\ell}}\right\|^{2} \\
& =(\ell !)^{2} \lim _{t \rightarrow 0} \frac{\|h(t)\|^{2}}{t^{2 \ell}} \\
& =(\ell !)^{2} \lim _{t \rightarrow 0} \frac{\left(\|h(t)\|^{2}\right)^{(2 \ell)}}{(2 \ell) !}=0 .
\end{aligned}
$$

Proof of Proposition 4.1. It suffices to prove this result for Lemma 3.1, for then the corresponding statement for Theorem 1.1 follows immediately (recall that, in the proof of Theorem 1.1, $\bar{f}$ is given by $\left(f_{1}, f_{2}\right)$, and $f_{1}$ is $C^{k+1}$; thus the regularity of $\bar{f}$ in Theorem 1.1 corresponds to that of $f_{2}$, which in turn coincides with the map $\bar{f}$ in Lemma 3.1). Next note that if $\gamma$ is disjoint from $\partial V$, then we are done, since $\bar{f}$ is $C^{2 \ell}$ on $M-\partial V$. Further, since $\gamma$ meets $\partial V$ transversally, it suffices to consider the curves $\gamma$ which map $(-1,0)$ to $V$ and $(0,1)$ to $M-\bar{V}$. Then $h:=\left(\partial_{i} \bar{f}\right) \circ \gamma$ 
vanishes on $(-1,0)$, is $C^{\ell}$ on $(0,1)$ and $\|h\|^{2}=g_{i i}(\gamma)$, which is $C^{2 \ell}$. So, by Lemma 4.2, $h$ is $C^{\ell}$. Next note that, since $\left(\partial_{i} \bar{f}\right) \circ \gamma$ are $C^{\ell}$, the Jacobian matrix of $f$ is $C^{\ell}$ on $\gamma$. Further, by the chain rule, $(\bar{f} \circ \gamma)^{\prime}(t)=\operatorname{Jac} \bar{f}_{\gamma(t)} \gamma^{\prime}(t)$. Thus, since $\gamma^{\prime}$ is $C^{2 \ell-1} \geq C^{\ell}$, it follows that $(\bar{f} \circ \gamma)^{\prime}$ is $C^{\ell}$, and therefore $\bar{f} \circ \gamma$ is $C^{\ell+1}$.

\section{Questions}

As we mentioned in the Introduction, we do not know if the isometric extension $\bar{f}$ in Theorem 1.1 can be constructed so that it is $C^{k}$ everywhere. The problem here is that we do not know whether $\bar{f}$ in Lemma 3.1 is $C^{k}$, or whether one can always choose $f$ in such a way that $\bar{f}$ will be $C^{k}$. More succinctly, we would like to know:

Question 5.1. Let $M$ be a $C^{\infty}$ manifold, and $g$ be a $C^{k}$ symmetric bilinear form on $M$ which vanishes on a set $B \subset M$ diffeomorphic to a closed ball, but is positive definite on $M-B$. Does there exist a $C^{k}$ mapping $f: M \rightarrow \mathbf{R}^{N}$ such that $f^{*}\langle\rangle=$,$g ,$ and $f$ is an embedding on $M-B$ ?

In other words, can one extend Nash's isometric embedding theorems to manifolds with degenerate metrics? We do not know the answer to this question even when $B$ is a point. If the answer to the above question is yes, then $\bar{f}$ in Theorem 1.1 is $C^{k}$.

\section{ACKNOWLEDGEMENT}

We thank Yuri Burago, Luis Florit, Ralph Howard, Misha Gromov, Howard Jacobowitz, Idjad Sabitov, David Spring, and Deane Yang for electronic communications related to this work.

\section{REFERENCES}

[1] S. Alexander, M. Ghomi, and J. Wong. Topology of Riemannian submanifolds with prescribed boundary, Duke Math. J., 152 (2010), 533-565.

[2] B. Andrews. Notes on the isometric embedding problem and the Nash-Moser implicit function theorem. In Surveys in analysis and operator theory (Canberra, 2001), volume 40 of Proc. Centre Math. Appl. Austral. Nat. Univ., pages 157-208. Austral. Nat. Univ., Canberra, 2002. MR:1953483 (2004c:53075)

[3] Y. Eliashberg and N. Mishachev. Introduction to the h-principle, volume 48 of Graduate Studies in Mathematics. American Mathematical Society, Providence, RI, 2002. MR.1909245 (2003g:53164)

[4] M. Ghomi. The problem of optimal smoothing for convex functions. Proc. Amer. Math. Soc., 130(8):2255-2259 (electronic), 2002. MR1896406 (2004d:26013)

[5] R. E. Greene. Isometric embeddings of Riemannian and pseudo-Riemannian manifolds. Memoirs of the American Mathematical Society, No. 97. American Mathematical Society, Providence, R.I., 1970. MR.0262980(41:7585)

[6] M. Gromov. Partial differential relations. Springer-Verlag, Berlin, 1986. MR864505 (90a:58201)

[7] M. Günther. Isometric embeddings of Riemannian manifolds. In Proceedings of the International Congress of Mathematicians, Vols. I, II (Kyoto, 1990), pages 1137-1143, Math. Soc. Japan, Tokyo, 1991. MR.1159298 (93b:53049)

[8] M. Günther. On the perturbation problem associated to isometric embeddings of Riemannian manifolds. Ann. Global Anal. Geom., 7(1):69-77, 1989. MR.1029846 (91a:58023)

[9] Q. Han and J.-X. Hong. Isometric embedding of Riemannian manifolds in Euclidean spaces, volume 130 of Mathematical Surveys and Monographs. American Mathematical Society, Providence, RI, 2006. MR2261749(2008e:53055) 
[10] N. J. Hicks. Notes on differential geometry. Van Nostrand Mathematical Studies, No. 3. D. Van Nostrand Co., Inc., Princeton, N.J.-Toronto-London, 1965. MR0179691 (31:3936)

[11] H. Jacobowitz. Extending isometric embeddings. J. Differential Geometry, 9:291-307, 1974. MR0377773 (51:13942)

[12] E. Kreyszig. Differential geometry. Dover Publications Inc., New York, 1991. Reprint of the 1963 edition. MR1118149(92d:53001)

[13] N. H. Kuiper. On $C^{1}$-isometric imbeddings. I, II. Nederl. Akad. Wetensch. Proc. Ser. A. 58 = Indag. Math., 17:545-556, 683-689, 1955. MR0075640(17:782c)

[14] J. Nash. $C^{1}$ isometric imbeddings. Ann. of Math. (2), 60:383-396, $1954 . \quad$ MR0065993 $(16: 515 \mathrm{e})$

[15] J. Nash. The imbedding problem for Riemannian manifolds. Ann. of Math. (2), 63:20-63, 1956. MR0075639 (17:782b)

[16] B. O'Neill. Elementary differential geometry. Elsevier/Academic Press, Amsterdam, second edition, 2006. MR2351345 (2008k:53001)

[17] A. V. Pogorelov. Extrinsic geometry of convex surfaces. American Mathematical Society, Providence, R.I., 1973. Translations of Mathematical Monographs, Vol. 35. MR.0346714 $(49: 11439)$

[18] I. K. Sabitov. Local theory of bendings of surfaces. In Geometry, III, volume 48 of Encyclopaedia Math. Sci., pages 179-256. Springer, Berlin, 1992. MR1306736

[19] M. Spivak. A comprehensive introduction to differential geometry. Vol. V. Publish or Perish Inc., Wilmington, Del., second edition, 1979. MR0532834 (82g:53003e)

School of Mathematics, Georgia Institute of Technology, Atlanta, Georgia 30332 E-mail address: ghomi@math.gatech.edu

$U R L$ : www . math.gatech.edu/ ghomi

Department of Mathematics, University of California, los Angeles, Los Angeles, CALIFORNIA 90095

E-mail address: greene@math.ucla.edu

$U R L$ : www.math.ucla.edu/ greene 